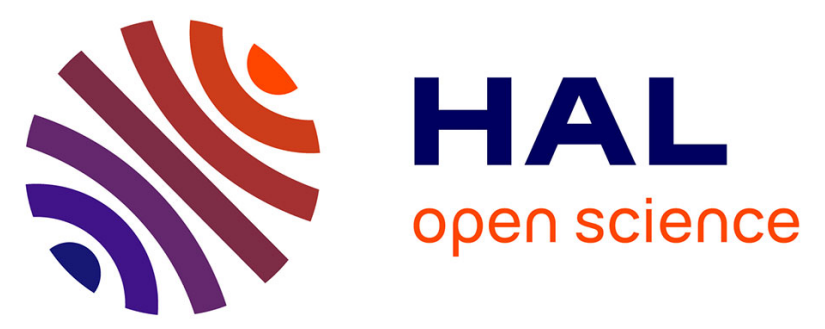

\title{
La structuration de la pratique du covoiturage en France: jeu d'acteurs et institutionnalisation
}

\author{
Stéphanie Vincent
}

\section{To cite this version:}

Stéphanie Vincent. La structuration de la pratique du covoiturage en France: jeu d'acteurs et institutionnalisation. ASRDLF, CRDT. Territoires et action publique territoriale : nouvelles ressources pour le développement régional - 45e colloque de l'ASRDLF, 25, 26 et 27 août 2008, Rimouski (UQAR), 2008, Rimouski, Canada. 12 p. halshs-00330357

\section{HAL Id: halshs-00330357 https://shs.hal.science/halshs-00330357}

Submitted on 14 Oct 2008

HAL is a multi-disciplinary open access archive for the deposit and dissemination of scientific research documents, whether they are published or not. The documents may come from teaching and research institutions in France or abroad, or from public or private research centers.
L'archive ouverte pluridisciplinaire HAL, est destinée au dépôt et à la diffusion de documents scientifiques de niveau recherche, publiés ou non, émanant des établissements d'enseignement et de recherche français ou étrangers, des laboratoires publics ou privés. 


\title{
TITRE : La structuration de la pratique du covoiturage en France : jeu d'acteurs et institutionnalisation
}

\author{
AUTEUR : Stéphanie VINCENT \\ LET I ENTPE \\ Rue Maurice Audin \\ 69518 Vaulx-en-Velin cedex \\ France \\ stephanie.vincent@entpe.fr
}

\section{THEMATIQUE : Transport et communication}

En France, le covoiturage semble être né avec la création de l'association Allostop en 1958 qui, la première, a mis en place un " autostop organisé »; le terme " covoiturage » ayant fait son apparition beaucoup plus tard, en 1989. A l'origine destinée à favoriser la mobilité de jeunes gens pas suffisamment fortunés pour s'offrir une voiture, la pratique a progressivement évolué pour davantage répondre aux enjeux de la congestion urbaine, du coût d'usage automobile et de la protection de l'environnement. Le covoiturage se trouvait alors en 2004 année de la réalisation de ce terrain ${ }^{1}$ - réapproprié par une multitude d'acteurs aux statuts et objectifs diversifiés. Du fait de cette diversité, les centres de décision se trouvent difficiles à repérer. Il s'agira alors ici dans un premier temps de décrire les acteurs du covoiturage en essayant de déterminer leur statut et leurs fonctions dans la promotion de cette pratique. Dans un second temps, l'analyse détaillée des acteurs permettra de mettre en lumière le jeu d'acteurs autour du covoiturage en France afin d'en dégager les forces et les faiblesses et ainsi d'évaluer les conditions de développement de cette pratique à l'échelle organisationnelle.

Le regard que nous adoptons ici se situe bien à l'échelle des organisations ou échelle méso-sociale (Desjeux, 2004) : nous nous intéressons donc aux organisations de covoiturage et à leur fonctionnement et non à la réappropriation de cette pratique par les usagers. De plus, dans le but d'évaluer le développement de cette pratique, le covoiturage est analysé à la manière du développement d'une innovation (Alter, 2002 ; 2003) d'où l'intérêt accordé aux acteurs qui la portent car «le développement [d'une innovation] ne peut être compris sans l'analyse sociologique des acteurs qui portent ce processus. » (Alter, 2002 : 7). Pour ce faire, nous avons adopté un regard inductif et compréhensif sur le fonctionnement des diverses structures de covoiturage afin d'en proposer une typologie. Nous avons ainsi travaillé à partir d'entretiens semi-directifs réalisés en face-à-face, au téléphone ou encore par email avec les responsables des structures de covoiturage. La recherche et la prise de contact avec ces dernières a principalement été réalisée grâce à Internet où chaque opérateur assure sa visibilité.

La méthode inductive utilisée a ainsi permis de reconstruire à partir du foisonnement des informations de terrain un premier cadre d'analyse distinguant cinq grands types de structures promouvant le covoiturage : projets émergeant de Plans de Mobilité (ou PDE ${ }^{2}$ ), sites de mises en relation fonctionnant exclusivement sur le Net, associations inscrites localement dans un territoire donné, opérateurs privés et enfin collectivités locales. L’analyse

\footnotetext{
${ }^{1}$ Les résultats présentés ici sur les organisations de covoiturage sont issus de nos travaux de thèse, soutenue en avril 2008. VINCENT S., Les altermobilités : analyse sociologique d'usages de déplacements alternatifs à la voiture individuelle. Des pratiques en émergence ? Université Paris-Descartes, France.

${ }^{2}$ Le Plan de Déplacements Entreprise est un ensemble de mesures visant à optimiser les déplacements liés au travail en favorisant l'usage des modes de transport alternatifs à la voiture individuelle, tels que la marche à pied, le vélo, les transports en commun, le covoiturage, les véhicules propres... Il s'agit d'une démarche volontaire menée par une entreprise, d'une administration ou d'un établissement public. (Définition disponible sur le site www.ademe.fr, consulté le 07 mars 2008).
} 
détaillée de ces acteurs permet alors de mettre au jour les contraintes de fonctionnement, essentiellement liées à la rareté des ressources financières auxquelles font face chacune de ces structures. Et ces contraintes de fonctionnement influencent fortement le jeu entre chacun des acteurs, mais aussi l'efficacité des services de mise en relation et par-là même le développement du covoiturage.

\section{1- Structuration du covoiturage en France : cinq grands types de d'organisations}

A partir des entretiens qualitatifs, cinq grands types de structures de covoiturage apparaissent. Parmi ces cinq types de structures, les projets de covoiturage montés dans le cadre de PDE ne se situent pas sur le même plan que les autres. En effet, ces projets ne s'adressent qu'aux usagers du site qui s'est investi dans la démarche du Plan de Déplacements ; les bases de données ne sont donc pas accessibles à tous mais réservées aux usagers du site, le plus souvent aux personnes qui y travaillent. Il existe ainsi une barrière à l'entrée du service; il n'est pas accessible à tous via un site Internet par exemple, mais réservé aux employés de l'entreprise, aux usagers d'un site précis, etc. De plus, comme nous le verrons ci-dessous, la réalisation des projets de covoiturage dans le cadre des PDE est bien souvent déléguée à un prestataire, association ou opérateur privé. C’est pourquoi nous nous concentrerons principalement sur les quatre autres types d'acteurs, même si les PDE constituent des initiatives favorables à la pratique du covoiturage.

\section{Des structures exclusivement virtuelles}

Le premier de ces quatre types d'acteurs est constitué de structures fonctionnant exclusivement sur Internet. Ces acteurs peuvent avoir deux types de statuts bien distincts. Dans certains cas, le site de covoiturage a un statut associatif ; ce statut possède l'avantage de pouvoir bénéficier de subventions. Dans d'autres cas, le site est propriété de son créateur ou de son racheteur. Précisons toutefois la réglementation française en matière de noms de domaine. Jusqu'au 20/06/06, un nom de domaine en «.fr » ne pouvait être détenu que par une société, une association ou un travailleur indépendant. C’était sur ce troisième statut qu'était détenu, par exemple, le site www.covoiturage.fr. Depuis le 20/06/06, n’importe quelle personne possédant une adresse postale en France peut créer son site en « .fr ».

Associés à une aucune autre forme d'existence concrète (telle que des locaux ou une ligne téléphonique), ces sites mettent à disposition des internautes un outil virtuel de mise en relation. Ils fonctionnent donc sur le mode du peer to peer: les utilisateurs de ces sites prennent ainsi directement contact entre eux pour organiser le partage d'un trajet. Si la consultation des trajets proposés est libre, l'utilisation du service est subordonnée à une inscription, relativement sommaire. Une fois inscrit, l'utilisateur peut proposer un nombre illimité de trajets et prendre contact avec d'autres utilisateurs du site. En pratique, les trajets saisis concernent plutôt des trajets uniques, c'est-à-dire qui n'ont lieu qu'une seule fois et sur de longues distances. Cette démarche est gratuite pour l'internaute, depuis le dépôt de trajet jusqu'à la prise de contact avec un covoitureur potentiel. L'unique échange financier concerne le dédommagement du conducteur par ses passagers au moment du trajet. Le service de mise en relation des covoitureurs par ce type de structures exclusivement virtuelle est donc totalement gratuit.

Etant donnée la gratuité du service, la mise en place d'un tel site n'est nullement rémunérateur pour son créateur. L'ensemble des coûts sont donc supportés par les créateurs et ceux-ci ne sont pas négligeables car pour attirer un maximum d'internautes, les interfaces sont améliorées régulièrement. La création de tels sites Internet se trouve alors essentiellement motivée par le militantisme. La plupart des responsables de ces sites exercent donc, en 
parallèle, une activité rémunérée quoique la plupart souhaiterait pouvoir vivre de leur site. Il se pose donc un problème de ressources financières. Même pour les sites ayant le statut associatif, les subventions obtenues se résument à des financements d'aide au lancement du site et ne perdurent pas dans le temps pour assurer un suivi. Afin de résoudre ce problème de ressources, plusieurs solutions ont été envisagées. La première consisterait à rendre le service de mise en relation ou l'inscription au site payant, mais elle se trouve être peu réalisable étant donnée la forte compétition qui règne entre les sites de covoiturage. Si l'un des sites devenait payant, les internautes se reporteraient sur les sites gratuits. De plus, cette stratégie tendrait à créer une barrière à l'accès au service qui risque d'entrer en contradiction avec l'objectif premier des militants qui est d'accroître le nombre d'utilisateurs du service, et par-là même celui des covoitureurs. Une autre stratégie évoquée par les responsables des sites serait de chercher un «tiers payant » qui assumerait le coût du service de mise en relation. Cette stratégie est déjà en partie en place grâce à la publicité et aux liens publicitaires sur les sites. D’autres tiers payants sont évoqués: entreprises, collectivités ou encore Etat, mais ces perspectives imaginées par certains restent encore loin d'aboutir à des stratégies concrètes.

\section{Des associations locales}

D’autres acteurs associatifs bénéficient pourtant de tels financements, notamment des associations qui cherchent à développer le covoiturage sur un territoire donné. Il s’agit ici du second type de structures repérées et que nous avons appelées les associations locales. Il en existe plusieurs sur le territoire français métropolitain, mais leur existence est fragile, car toujours fortement dépendante des subventions dont elles font l'objet. Ces structures sont de nature associatives ; elles disposent comme les précédentes d'un site Internet qui assurent leur visibilité mais elles ne s'y limitent pas. Elles construisent également leur action à partir de structures locales : elles possèdent donc des locaux permettant non seulement un accueil humain, mais aussi téléphonique. De plus, contrairement aux sites fonctionnant exclusivement de manière virtuelle, les associations locales de covoiturage effectuent un véritable travail de mise en relation entre les covoitureurs. Enfin, troisième différence avec les sites présentés cidessus, les équipages ne sont pas essentiellement formés pour des trajets uniques et de longue distance. A l'inverse, le travail de la plupart de ces associations a pour objectif de favoriser des covoiturages de courte distance, sur des trajets quotidiens tels que les trajets domiciletravail. Certaines ont même mis en place un double service : elles mettent en relation des covoitureurs d'une part pour des trajets uniques, souvent de longue distance et d'autre part pour des trajets plus quotidiens de type domicile-travail.

Leurs objectifs consistent bien alors à rechercher une diminution du nombre de véhicules en circulation afin, d'une part, de limiter la congestion et, d'autre part, la pollution urbaine locale. L'intérêt des associations locales à monter de tels projets de covoiturage consiste également en un intérêt militant. Néanmoins, leurs services ne sont pas entièrement gratuits mais elles demandent une petite participation financière pour le travail de mise en relation qu'elles opèrent, tantôt sous forme de cotisation annuelle, tantôt sous forme de forfaits de mise en relation. Pour les trajets quotidiens et répétés tels que les trajets domicile-travail, la cotisation n'est souvent demandée qu'une fois que les équipages de covoitureurs sont constitués et fonctionnent. Un tel dispositif pose bien évidemment des problèmes de fidélisation du service. Une fois mis en relation, les covoitureurs peuvent toujours échapper au financement du service qu'est la cotisation annuelle. Ainsi, comme pour les sites de covoiturage exclusivement virtuels, des problèmes de ressources financières se posent.

Les associations locales ont ainsi déployé diverses stratégies pour pallier ce problème de ressources. En tant qu'associations localisées, elles peuvent bénéficier de subventions qui se justifient principalement dans deux domaines : d'une part, l'environnement et d'autre part 
l'insertion sociale. Des subventions d'ordre environnemental sont effectivement accordées aux associations de covoiturage dans la mesure où elles œuvrent dans le sens d'une réduction du nombre de voiture, donc de la pollution automobile associée. Dans une perspective d'insertion sociale, les associations de covoiturage permettent également l'accès à l'automobilité à une population défavorisée en vue de sa réinsertion sociale. Ces financements proviennent alors de l'Etat, notamment au travers de l'ADEME ${ }^{3}$, des collectivités locales (région, département) ou encore de fonds européens.

Depuis peu, ces associations ont mis en place diverses stratégies pour garantir leurs ressources. Ces stratégies visent tout d'abord à fidéliser leurs adhérents grâce à des avantages offerts aux adhérents à jour de leur cotisation. Parmi ces avantages, le taxi-joker ou garantie de retour permet aux covoitureurs de bénéficier d'une solution de retour gratuite (transports en commun, autre covoitureur ou taxi) en cas de défection du covoitureur habituel. En dehors de ces avantages comparatifs, la fidélisation des adhérents sur la seule mise en relation reste difficile car le service est peu perçu par les usagers. D’autant que le paiement du service de mise en relation rentre en contradiction avec la gratuité des sites virtuels. Les associations locales cherchent donc, en parallèle, à développer des partenariats durables avec leurs financeurs de départ, par exemple, la ou les communautés d'agglomérations sur lesquelles elles sont implantées. C'est par exemple le cas de Covoituval qui a réussi à établir un partenariat avec le SICOVAL, communauté d'agglomération située au sud-est de Toulouse, sur laquelle l'association est implantée. Enfin, un troisième type de stratégies vise plutôt des organisations (entreprises, collectivités...) qui sont démarchées afin de recruter toujours plus de covoitureurs. Cette stratégie plus incisive a été notamment adoptée par ALLOSTOP Rennes dans son volet de covoiturage quotidien et rencontre d'assez bons résultats. Elle possède l'avantage majeur de faire assumer par l'organisation le coût de mise en relation des équipages.

Ainsi, les associations cherchent à mettre en place des modes de financements novateurs. Par le biais de ces démarchages, la source financière des associations de covoiturage ne réside plus seulement dans des subventions, mais glisse progressivement du côté des organisations qui assument, du moins en partie, la charge financière du service de covoiturage pour leurs salariés. Ainsi, les associations locales de covoiturage assurent la fonction d'opérateur du covoiturage pour des organisations, ce qui les place en concurrence directe avec un autre type d’acteur structurant du covoiturage : les opérateurs privés de covoiturage.

\section{Des opérateurs privés de covoiturage}

En effet, il existe aujourd'hui plusieurs opérateurs privés de covoiturage en France, c'est-àdire des sociétés qui se chargent de mettre en œuvre le covoiturage pour des entreprises, administrations ou établissements publics. Du point de vue juridique, il ne s'agit donc plus ici de structures associatives mais d'entreprises. Une récente étude du CERTU (CERTU, 2007) en a ainsi identifié plusieurs (voir schéma 1).

L'opérateur de covoiturage se trouve mandaté par une entreprise, une administration ou une collectivité locale afin d'organiser un covoiturage sur son site. Ces organisations délèguent la réalisation de leur projet de covoiturage à un opérateur privé essentiellement du fait que ces derniers apparaissent comme des experts du covoiturage. L'opérateur privé met ainsi à disposition de l'organisation mandataire ses compétences sur le sujet, une base de données, un logiciel cartographique et une interface pour les utilisateurs du service. Or, ces outils sont à la fois difficiles et longs à construire ; s'adresser à un opérateur allège alors en temps, en difficulté et en coût les organisations qui souhaitent réaliser un covoiturage. De

\footnotetext{
${ }^{3}$ ADEME (Agence de l'environnement et la maitrise de l'énergie) est une agence publique qui finance un grand nombre de projets et d'initiatives en faveur de l'environnement.
} 
plus, les outils proposés par les opérateurs, notamment les outils cartographiques, s'avèrent plus élaborés que ceux que pourraient créer l'entreprise ou l'administration concernée.

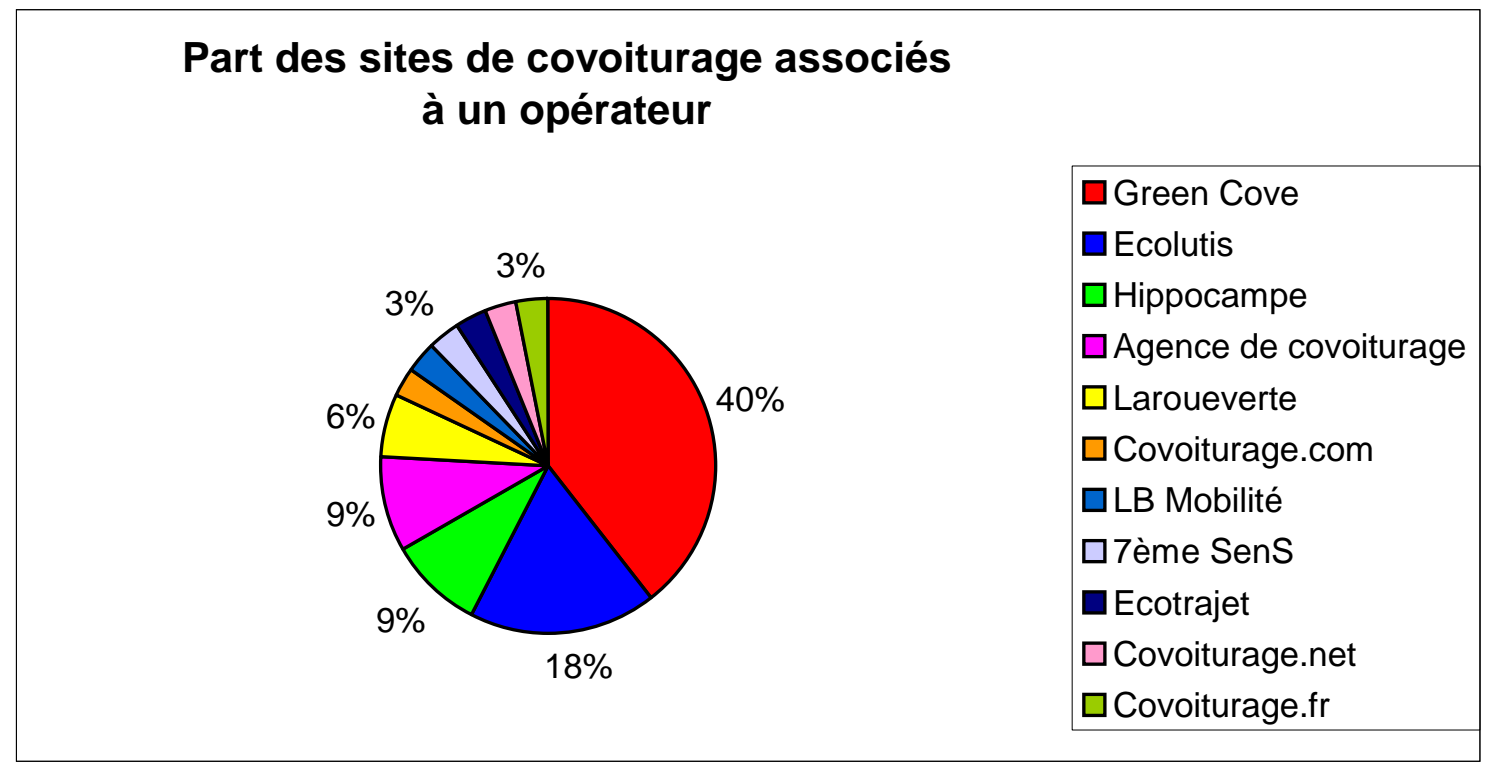

Figure 1- Part des sites de covoiturage associés à un opérateur

Suite à plusieurs entretiens réalisés auprès d'opérateurs de covoiturage ${ }^{4}$, nous avons pu élaborer un modèle expliquant leur mode de fonctionnement. L’objectif des opérateurs est de mutualiser au maximum leurs bases de données car, plus l'offre est grande, plus il y a de chances de la faire correspondre avec une autre. Ainsi, certains opérateurs associent même un site gratuit fonctionnant virtuellement à un service payant proposé aux entreprises et collectivités. De ce fait, les bases d'offres de trajet peuvent être fusionnées, le site gratuit fournissant une base de données déjà conséquente qui renforce l'efficacité du service offert aux entreprises et collectivités. Même si ces deux services se trouvent indépendants l'un de l'autre, ils s'enrichissent mutuellement puisqu'ils partagent une même base de trajets. Cette stratégie permet de proposer à l'organisation mandataire d'un projet de covoiturage, une base de données d'ores et déjà remplie d'annonces, ce qui constitue un avantage considérable.

Du point de vue économique, les opérateurs de covoiturage ont choisi de faire financer le service de covoiturage, non par les usagers du service, mais par les organisations qui les emploient. Pour les organisations, le coût de la mise en place d'un service de covoiturage est principalement supporté à l'entrée, c'est-à-dire au moment de la création du service. En effet, l'entretien et le suivi du site restent peu coûteux, d'autant qu'il peut être réalisé de manière autonome par l'organisation mandataire du service : dans ce cas, l’opérateur privé ne conserve qu'un rôle de modérateur.

Les opérateurs de covoiturage font vivre leur entreprise grâce à la vente de leurs services auprès d'entreprises, d'administrations ou de collectivités locales. Ainsi, l'intérêt des opérateurs privés de covoiturage reste un intérêt financier à voir se développer des projets de covoiturage. Néanmoins, le fait d'avoir adopté un tel modèle économique pose deux questions. Tout d'abord, en regard des autres acteurs précédemment décrits, les opérateurs privés de covoiturage se trouvent non seulement en concurrence entre eux mais aussi avec les associations locales de covoiturage. En effet, elles aussi ont développé une expertise autour de la mise en place de covoiturage ; certaines d'entre elles ont même adopté une stratégie de

\footnotetext{
${ }^{4}$ Dont nous tairons les noms, par souci de confidentialité.
} 
démarchage d'entreprises ou d'administrations sur des zones d'emploi données. Opérateurs de covoiturage et associations se trouvent donc en concurrence alors même que leurs intérêts divergent.

La seconde question soulevée par l'adoption de ce modèle économique concerne la fidélisation des clients. En effet, les opérateurs sont amenés à fidéliser leurs clients, d'autant plus que le milieu s'avère concurrentiel. Ainsi, les opérateurs interrogés nous ont fait part des différentes stratégies mises en place dans ce but. Ils cherchent, par exemple, au travers de labellisation, à rassurer leurs clients sur la qualité et l'efficacité du service. La fidélisation fait également intervenir la valorisation de l'expertise constituée par l'opérateur sur la question du covoiturage qui promet au client un service rapidement opérationnel et efficace. Enfin, et c'est le principal argument des opérateurs, la fidélisation des clients se joue sur la performance du service permise par la mutualisation des bases de données.

Les clients de ces opérateurs de service sont constitués principalement de deux types d'organisations : d'une part, des entreprises, administrations et établissements publics qui mettent en place un covoiturage dans le cadre d'un PDE ; d'autre part, des collectivités locales, communes, intercommunalités, départements voire régions pour lesquels la réalisation d'un covoiturage constitue un volet de leur agenda $21^{5}$. Néanmoins, la contrainte majeure au développement de tels services de covoiturage réside dans le fait que la réalisation d'un PDE ou d'un agenda 21 relève en France simplement de l'incitation et non de l’obligation légale 6 .

\section{Des acteurs publics institutionnels}

L’évocation de la démarche Agenda 21 nous amène ainsi à évoquer le quatrième type de structure promouvant le covoiturage en France : il s'agit d'acteurs publics institutionnels. Les acteurs concernés peuvent être tant des communes, des intercommunalités, des départements ou des régions, c'est-à-dire tous les échelons de gouvernance pour lesquels la réalisation d'un agenda 21 est possible. Pour les acteurs publics institutionnels, l'intérêt à développer des projets de covoiturage réside essentiellement dans un intérêt d’ordre général, celui de se conformer aux grands engagements de la France au niveau international. C'est pourquoi les projets s'inscrivent principalement dans le cadre d'agendas 21.

Nous avons ainsi pu spécifiquement observer l'implication d'acteurs publics dans la promotion du covoiturage au niveau départemental, dans le Finistère. En effet, le département du Finistère fut le premier à réaliser un projet de covoiturage à l'échelle du département. Cette initiative se trouve être relativement originale car, en France, le département n'a pas de compétences en matière de gestion des déplacements. Le projet se fonde sur l'observation préalable d'une pratique préexistante. Ainsi, les agents avaient remarqué des voitures stationnées près des échangeurs d'autoroute, témoignant de l'existence de covoiturages informels et spontanés. Mais ces stationnements posaient des problèmes de sécurité. Ainsi, quoique le département n'ait pas de compétences en déplacements, il s’est lancé dans la construction d'aires de covoiturage au niveau de ces échangeurs d'autoroute dans le but de faciliter et de sécuriser les stationnements dans ces zones.

\footnotetext{
${ }^{5}$ En 1992, le Sommet de la Terre à Rio de Janeiro a donné lieu à la rédaction d’un programme appelé Agenda 21 ou Agenda pour le $21^{\text {ème }}$ siècle. Chacun des Etats présents au Sommet et ayant signé ce programme s'est alors engagé à mettre en place une Commission Nationale du développement durable dont le rôle est d'encadrer la réalisation d’Agendas 21 locaux. En France, les Agendas 21 peuvent être réalisés par tout type de collectivité locale. Il s'agit pour elles de mettre en place un ensemble de mesures favorables au développement durables. Ainsi, un certain nombre de ces mesures concernent la gestion durable des déplacements.

${ }^{6}$ Les Agendas 21 constituent des démarches entièrement volontaires de la part des collectivités locales. Quant aux PDE, les entreprises, administrations et établissements publics sont légalement incités à en mettre en place au travers du Plan Climat (2004).
} 
Le cœur du projet reste, cependant, constitué par le lancement, en septembre 2005, d'un site Internet de mise en relation de covoitureurs. Le site propose, dans ce but, une cartographie du département et des départements limitrophes afin que l'utilisateur puisse visualiser son trajet. Mais, à la différence des autres sites Internet de covoiturage, ce dernier s'adresse prioritairement au trajets locaux ; ainsi, les trajets saisis sur le site doivent posséder au moins une extrémité (origine/destination) dans le Finistère ${ }^{7}$. Un projet d'association avec les départements limitrophes semble être en discussion, notamment avec le Morbihan et les Côtes d'Armor, dans la perspective d'une mutualisation des données.

Ce projet de covoiturage départemental possède trois volets : d'abord, la mise en relation d'équipiers au travers du site, la création d'aires de covoiturage et enfin, un troisième volet essentiel aux yeux du responsable de projet : la communication, au travers du site Internet mais aussi par des campagnes d'informations destinées aux habitants du département (campagnes d'affichage, par exemple). Pour ce quatrième type d'acteur du covoiturage, la question des ressources se pose de manière moins nette que pour les précédents. En effet, il se trouve financé d'une part, par les fonds propres du département et, d'autre part, par une subvention de l'Ademe à la réalisation d'un Agenda 21.

Les acteurs autour du covoiturage se trouvent être de nature et de fonctionnement très divers. Ainsi, cinq types d'acteurs se partagent le "marché » du covoiturage : des sites de particuliers, des sites associatifs, des associations possédant une assise locale, des opérateurs priés et enfin des acteurs publics institutionnels tels que les collectivités locales. La description du fonctionnement de chacun de ces acteurs laisse apparaître un élément fortement structurant du jeu d'acteurs : la rareté des ressources. Ainsi, les sites exclusivement virtuels ne possèdent pas de financements exceptés leurs fonds propres et doivent renoncer à faire payer le service de mise en relation aux utilisateurs. Les associations locales mettent en place des alliances et partenariats avec d'autres acteurs, notamment les collectivités locales des territoires sur lesquels elles travaillent ou encore les entreprises et administrations dont les personnels peuvent être intéressés par le service. Les opérateurs de covoiturage rencontrent les mêmes limites de financements et mettent en place des stratégies similaires. Au final, seuls les projets montés par les acteurs publics institutionnels ont accès à des financements relativement durables dans la mesure où leur action entre en coïncidence avec un intérêt général environnemental conforme aux engagements internationaux de la France. En dehors des acteurs publics institutionnels, l'ensemble des acteurs du covoiturage tirent leurs financements de trois sources: l'Union Européenne, L'Etat français au travers des subventions de l'Ademe ou bien, de manière décentralisée, des collectivités locales et enfin des entreprises, administrations et établissements publics susceptibles de réaliser des PDE sachant que ces derniers peuvent également bénéficier de financements de l'Ademe pour monter leurs projets de PDE. Une telle rareté des ressources n'est pas sans conséquence sur le fonctionnement du jeu d'acteurs qui se révèle fortement concurrentiel mais aussi de l’efficacité du service.

\footnotetext{
${ }^{7}$ D’après les premières études sur le site et sa fréquentation, réalisées en juin 2006, 90\% des inscrits résident dans le département du Finistère.
} 


\section{2- Conséquences de la rareté des ressources de financements sur la pratique et son développement}

Dans la perspective de l'analyse du développement du covoiturage à la manière d'une innovation, la diversité des acteurs repérés autour du covoiturage pourrait laisser penser que le covoiturage serait dans la seconde phase de son institutionnalisation. En effet, l'innovation se définit comme le processus social qui amène une invention, c'est-à-dire un objet ou une pratique nouvelle, à devenir la nouvelle norme d'usage. Pour ce faire, l'innovation passe par plusieurs phases de développement qui peut être formalisé sous la forme d'une courbe en $S$ : " peu d'usages au départ avec seulement quelques pionniers ; beaucoup d'usages ensuite avec les imitateurs, et de nouveau peu d'usages à la fin, ou parce que le marché est saturé, ou parce qu'il touche les 'réfractaires'. » (Alter, 2006 : 269). Ainsi, la diversité des acteurs gravitant autour du covoiturage laisserait à penser que ce dernier est dans la seconde phase de son développement, c'est-à-dire celle durant laquelle les pionniers sont rejoints par des essaims d'imitateurs. Cependant, l'analyse du mode de fonctionnement des différents acteurs montre les difficultés de financements rencontrées par ces derniers. Or, ces deux éléments apparaissent contradictoires et il convient alors de proposer une synthèse sur la place du covoiturage aujourd'hui en France : est-ce bel et bien une pratique en émergence ? ou bien s'agit-il encore de projets militants qui restent à la marge ? Et si tel est le cas, quelles pourraient être les conditions permettant le développement du covoiturage ?

La rareté des ressources à laquelle se confrontent les acteurs du covoiturage tend à induire deux conséquences. Tout d'abord, elle occasionne une forte incertitude relative à la pérennité des financements. Cette incertitude amène alors une forte concurrence entre les acteurs, chacun cherchant à protéger son projet et ses propres ressources. Deuxièmement, la rareté des ressources met en exergue les difficultés financières ressenties par la majorité des acteurs du covoiturage pour faire vivre leur projet. En effet, la réalisation d'un projet de covoiturage s'avère souvent longue, difficile et peu rémunératrice. Pourquoi les associations, les particuliers ou les opérateurs privés continuent-ils alors ? Il semblerait que la réalisation de tels projets soit moins liée à la perspective d’une activité rémunératrice qu'à un engagement personnel militant.

\section{Des difficultés d'alliance nuisibles à l'efficacité du service}

L’analyse détaillée précédente montre que la rareté des ressources constitue un élément structurant du jeu d'acteurs autour du covoiturage, en grande partie car le financement constitue une incertitude majeure pour les acteurs. Cette incertitude vient alors s'ajouter à d'autres incertitudes directement liée à la réalisation d'un covoiturage (trouver des personnes désireuses de covoiturer, coïncidence des itinéraires et des horaires). Mais, ces incertitudes relatives à la coïncidence des offres de trajets diminuent avec l'augmentation du nombre d'offres : plus il y a d'offres de trajets, plus il y a de chances que deux offres coïncident.

Ainsi, l'une des possibilités pour limiter les incertitudes précédemment décrites consisterait à mutualiser les offres de trajets. Mais, ces offres se partagent entre différents opérateurs : une mutualisation des offres ne serait alors possible que dans la mesure où les acteurs s'allieraient pour partager leurs connaissances sur le covoiturage. Or, étant donné la rareté des ressources financières attribuées aux projets de covoiturage, chacun des acteurs cherche à conserver le pouvoir qu'il possède sur les autres en évitant de partager le nom de ses clients - donc de ses sources financières. On observe alors une volonté, de la part de chacun des acteurs, de créer et conserver des «zones d'incertitude » (Crozier, Friedberg, 1977) qui lui confère alors du pouvoir sur les autres. Ces zones d'incertitudes consistent 
essentiellement à ne pas divulguer aux autres le nom des clients et à ne pas partager les offres de trajets ${ }^{8}$.

Ce refus de partager des informations engendre alors un effet pervers pour l'ensemble des acteurs et pour la réussite de leurs projets : la compétition entre les acteurs du covoiturage est donc un jeu à somme négative. Elle entraîne, en effet, une dispersion de l'offre qui nuit à l'efficacité globale du service. Cet effet indésirable est notamment soulignée par L. Lancart, responsable des politiques de déplacements urbains à l'ADEME : "On s'y perd et ça ne marche pas. Il y a trop de sites et pas assez de visiteurs sur chacun. Il faudrait massifier, fédérer tout ça » (cité dans la presse régionale, Ouest France, 21 juillet 2007). De plus, du point de vue de l'utilisateur, cette dispersion de l'offre tend à induire une complexification du choix. En effet, si l'on se réfère à la théorie de la rationalité limitée de $\mathrm{H}$. Simon, il s'avère que les décisions se prennent toujours en l'absence de la totalité des informations disponibles, une telle omniscience du décideur s'avérant impossible. Mais, choisir implique de pouvoir comparer les différentes offres; or, plus ces dernières s'avèrent nombreuses plus le choix risque d'être difficile (Simon, 1997). Nous pouvons alors avancer ici l'hypothèse que l'usager, face à une telle offre, se trouve découragé ou, à l'inverse, multiplie les ressources afin de garantir la réalisation de son trajet. Cette seconde hypothèse amène alors à reconsidérer le nombre de trajets saisis sur chaque site de covoiturage. Ils sont effectivement nombreux, mais certainement redondants d'un site à l'autre...

Ainsi, de manière indirecte, la rareté des ressources implique une forte concurrence entre les acteurs, et donc une impossibilité à créer des « alliances » entre eux (Callon, 1986 : 185). Chacun cherche alors à établir des partenariats financiers à moyen terme avec des intercommunalités, des entreprises ou encore des syndicats de transport. Partageant les mêmes stratégies partenariales, ces opérateurs se trouvent alors en concurrence ce qui limite d'autant les marges de manœuvre de chacun. Les échanges entre acteurs s'avèrent rares, les partages d'informations limités, celui des bases de données impossibles : le jeu se trouve fortement concurrentiel. L'efficacité globale des mises en relation en pâtit, limitant par-là même le développement de l'usage.

\section{A l'origine des projets : des engagements militants et passionnés}

Les projets de covoiturage se heurtent donc à la rareté des ressources ; de ce fait, ils restent peu rémunérateurs pour leurs créateurs. Cette limite pose alors la question des motivations à monter un projet de covoiturage mais aussi à le faire perdurer. La description des acteurs et l'évocation de leur intérêt à développer le covoiturage laisse alors entrevoir une réponse à cette question. En effet, que ce soit les sites de covoiturage de particuliers, les sites associatifs, ou les associations locales, toutes ces structures fonctionnent sur la base du militantisme - y compris les opérateurs privés de covoiturage. Pour mieux comprendre cette dimension militante des projets, un changement de focale s'opère ici. Les acteurs ont été précédemment décrits à l'échelle méso-sociale (Desjeux, 2004 : 4) ; il s’agira ici non plus de s'intéresser à la dimension organisationnelle des projets de covoiturage mais aux parcours biographiques de ceux qui les portent.

Les opérateurs privés de covoiturage apparaissent au premier abord principalement intéressés par la réussite financière de leur projet : ils montent des entreprises organisatrices de covoiturage afin d'en tirer profit. Pourtant, lors des entretiens avec ces entrepreneurs, il est apparu que tout ne se joue pas pour autant sur le mode économique. L'origine des entreprises de covoiturage trouve elle aussi sa source dans les méandres de l'engagement personnel au

\footnotetext{
${ }^{8}$ Ce refus de mutualiser les offres s'illustre notamment dans l'impossibilité à fédérer les différentes initiatives en faveur du covoiturage. Cette difficulté fut soulignée par plusieurs acteurs du covoiturage durant les interviews. Certains évoquaient même l’idée d'une crainte de la concurrence.
} 
profit d'un bien commun et de l’intérêt général : diminution de la congestion, diminution de la pollution locale liée à l’automobile, etc.

Du fait de la recherche de cet intérêt général, dépassant les intérêts particuliers, les personnes à l'origine des projets de covoiturage se révèlent alors être des militants au sens où J. Ion, S. Franguiakis, et P. Viot, dans Militer aujourd'hui, définissent ce terme : ils agissent pragmatiquement pour «apporter (...) une réponse immédiate, même provisoire, aux problèmes considérés » (2005 : 7). Comme l'expliquent ces auteurs, "Il s’agit dans tous les cas de se prémunir ou de conjurer de nouveaux risques majeurs (le saccage de la planète, les tensions internationales, l'opposition Nord/sud, la misère ici et ailleurs). L'objectif est moins de se battre pour des lendemains meilleurs que de conjurer des dangers imminents. » (Ion et alii, 2005 : 12). J. Ion parle alors d'un «idéalisme pragmatique » (Ion, 2005, p 96). Ainsi, si certains semblent avoir des idéaux écologistes de long terme, tels que la préservation de l'environnement au sens large, leur action porte néanmoins hic et nunc, notamment sur la diminution de la pollution urbaine environnante et les émissions de $\mathrm{CO}^{2}$.

Au-delà de cet arrière-plan militant, de forts imaginaires sous-tendent la création des sites Internet de covoiturage. En tant qu'anciens usagers du covoiturage ou de l'autostop, ils ont développé un imaginaire enchanté voire utopiste autour de la pratique du covoiturage et cet imaginaire intervient comme un moteur dans la réalisation du projet (Desjeux, 2004 : 32-34). Ainsi, l'action des créateurs de sites Internet se trouve guidée par des idéaux et imaginaires autour de la résolution des problèmes liés à la mobilité. Par leur action, ils rentrent alors dans l'engagement militant. Mais, cet engagement de départ ne risque t-il pas de s'essouffler avec le temps s’il n’est pas relayé par une compensation économique?

Il semblerait que non : la temporalité des projets s’inscrit dans le long terme. Ainsi, loin de faire long feu quelques mois après leur lancement, les projets de covoiturage se trouvent suivis et investis dans la durée par leurs créateurs. Il est vrai que ces projets constituent une activité à part entière pour certains, par exemple pour les entrepreneurs de sociétés de service de covoiturage. Mais, la place du projet est différente pour les responsables de sites dont l'existence n'est que virtuelle ou encore pour les responsables des associations locales. Etant donné que ces projets ne sont pas rémunérateurs, ils constituent, pour certains une activité hors travail. Dans d'autres cas, les militants du covoiturage ne travaillent pas ou plus et s'investissent donc à temps complet dans leur projet. Mais, dans tous les cas, la réalisation d'un projet de covoiturage ne peut se décrire simplement comme un loisir. La régularité de l'investissement du créateur, la durabilité des engagements dans le projet se trouvent être caractéristiques des " passions ordinaires » mises en lumière par C. Bromberger (Bromberger, 1998). D’autres éléments nous laissent deviner cette dimension passionnelle, par exemple l'investissement en temps et en argent des militants dans leurs projets ; " [le passionné] c'est quelqu'un qui paye de sa personne et ne lésine pas sur les investissements - en argent, en temps, en équipements divers - nécessaires à la réalisation du projet qui le taraude » (Bromberger, 1998 : 27). Et, à l'inverse, ceux qui ne sont pas suffisamment passionnés abandonnent très rapidement.

Entre engagement militant et passion, la réalisation d'un projet de covoiturage (associatif, virtuel ou entrepreneurial) reste également l'incarnation du travail de son créateur. Ainsi, les responsables de projets de covoiturage s'apparentent alors à ce que N. Alter appelle, en référence à Schumpeter, l' "entrepreneur-innovateur». Nous retrouvons du reste, dans ce concept, la dimension passionnelle de cette activité : les «entrepreneurs-innovateurs » n’agissent pas selon la logique de la rationalité économique mais sont animés par « la passion, plus que la raison» (Alter, 2003 : 26). Mais aussi l'engagement militant dans un temps présent : «l'entrepreneur-innovateur représente tout d'abord un personnage plus intéressé par 
l'action elle-même, par l'engagement qu'elle suppose, que par les bénéfices financiers qu'il en tire, ou des plaisirs qu'il peut tirer de la jouissance de ces bénéfices » (Alter, 2003 : 26). Une double perspective temporelle est donc à prendre en compte ici : si l'action militante porte bien sur le temps présent, les projets militants s'inscrivent quant à eux dans la durée. Ainsi, d'une part, les actions s'inscrivent dans le court terme au sens où elles visent la résolution de problèmes ici et maintenant ; d'autre part, les projets de covoiturage s'inscrivent dans le long terme puisqu'ils font l'objet d’un suivi régulier dans le temps.

La rareté des ressources financières à laquelle font face les opérateurs de covoiturage constitue un frein au développement de l'usage. Les difficultés financières entraînent notamment un repli des acteurs sur eux-mêmes et un refus de partager leurs informations et d'unir leurs bases de trajets. Pourtant, la mise en commun des offres permettrait certainement un plus grand nombre de mises en relation, donc une plus grande efficacité du service. De plus, la rareté des ressources financières rend la mise en œuvre de projets de covoiturage difficile et peu rémunératrice ; de ce fait, cette activité reste principalement aux mains de militants et de passionnés qui cherchent à servir l’intérêt général au travers de leur action. Ainsi, il semble y avoir principalement deux modèles qui président à la réalisation de covoiturage. Le modèle principal reste, comme nous l'avons signalé, un modèle militant, que ce soit pour les associations de covoiturage ou encore les opérateurs privés. Mais, pour les acteurs publics institutionnels, la réalisation de covoiturage semble davantage obéir à un intérêt général, celui de participer à la réduction des gaz à effet de serre et ainsi de se conformer aux grands engagements de la France sur la scène internationale.

Ainsi, on peut observer une multitude d'acteurs autour du covoiturage qui laisse à penser que le développement du covoiturage serait dans une phase d'imitation, celle durant laquelle « des essaims d'imitateurs [qui] reproduisent et aménagent les innovations, créant des grappes d'innovations secondaires » (Alter, 2003 : 14). Pourtant, plusieurs autres indices relevés dans l'analyse détaillée des acteurs et de leur fonctionnement montrent, à l'inverse, que le covoiturage ne se trouve qu'au tout début de la courbe d'innovation. Tout d'abord, le covoiturage reste une activité non rémunératrice, dont le développement se trouve essentiellement encore aujourd'hui porté par des militants. Or, les militants constituent la base du business modèle lorsqu'une activité n'est pas rentable. Ainsi, ces derniers ont besoin de mobiliser imaginaires et affectifs pour s'engager dans leurs projets. Au final, le covoiturage organisé ne semble pouvoir se développer véritablement que lorsque l'activité deviendra davantage rémunératrice pour ses organisateurs. Néanmoins, le seul développement de structures organisant le covoiturage ne suffit pas à conclure au développement ou nondéveloppement de la pratique du covoiturage car le covoiturage constitue aussi et surtout une pratique informelle entre au moins deux interconnaissances et ne nécessite donc pas obligatoirement le passage par des instances de mise en relation. 


\section{Bibliographie :}

ADEME, 2003 (octobre), « Réaliser un Plan de déplacements entreprise », Ademe Editions. ADEME, ATEMA Conseil, MHC Conseil, 2005, Evaluation nationale des Plans de Déplacements Entreprise, disponible en ligne sur le site www.plan-deplacements.fr Alter N., 2002, «L'innovation: un processus collectif ambigu », in Alter N. (dir.) Les logiques de l'innovation, Paris, La Découverte.

Alter N., 2003 [2000], L'innovation ordinaire, Paris PUF.

Alter N. (dir) 2006, Sociologie du monde du travail, Paris, PUF.

Bromberger M., 1998, Passions ordinaires : du match de football au concours de dictée, Paris, Bayard.

Callon M., 1986, «Eléments pour une sociologie de la traduction : la domestication des coquilles Saint-Jacques et des marins-pêcheurs dans la baie de Saint-Brieuc », in L'année sociologique, $\mathrm{n}^{\circ} 36, \mathrm{pp} .169-208$.

CERTU, 2007 (octobre), Le covoiturage en France et en Europe : état des lieux et perspectives, Rapport de recherche, Lyon, CERTU.

Crozier M., Friedberg E., 1977, L'acteur et le système, Paris, Seuil.

Desjeux D., 2004, Les sciences sociales, Paris, PUF.

Ion J., 2005, "Individualisation et engagement public » in CORCUFF, P., ION, J., de SINGLY, F., Politiques de l'individualisme, entre sociologie et philosophie, Paris, éditions Textuel, collection la Discorde, pp 88-112.

Ion J., Franguiadakis S., Viot P., 2005, Militer aujourd'hui, Paris, Autrement.

Simon H. A., 1997 , Models of Bounded Rationality: Empirically Grounded Economic Reason, vol. 3, Massachusetts Institute of Technology Press. 